\title{
P 175 EVALUATING A PROFESSIONAL DEVELOPMENT PROGRAMME
}

Christine Hedges, ${ }^{1,2}$ Bee Wee ${ }^{1,2}$. 'Sobell House Hospice, Oxford, UK; ${ }^{2}$ University of Oxford

10.1136/bmjspcare-2014-000654.216

Background \& Aims This project used the Kirkpatrick ${ }^{\mathrm{TM}}$ model of learning to help evaluate a Senior Nurses' Professional Development Programme (PDP). It acknowledges that the educational impact on practice, both directly and indirectly, is difficult to measure and is in need of development. In light of this, we aimed to use the Kirkpatrick learning framework to consider a more comprehensive evaluation.

Evaluation tools Kirkpatrick's model identifies 4 levels/stages of learning which assist an evaluation. We concentrated on levels 2 and 3.

Level 2 - Evaluation of trainee learning: Likert scales quantified increases in topic understanding and confidence levels.

Level 3 - Evaluation of trainee Behaviour: A focus group gained feedback on changes in practice.

Results Level 2 - Evaluating evidence of learning -

We were able to present increases in topic related learning and confidence, identifying differences.

Level 3 - Evaluating changes in practice -

Changes in practice emerged in two separate ways. Firstly, changes resulted from the programme's topics of education, critical appraisal and research design, pain management and leadership/time management. Secondly, benefits emerged from having attended the programme itself. There were increases in; commitment to practice development, group cohesion, positive working practices between teams, a valuing of and commitment to the ethos and style of the PDP itself as a model for education and training.

Conclusions The Kirkpatrick model provides a sound structure for training evaluation. Using quantitative and qualitative methods, were able to: quantify positive increases in understanding and confidence in the session topics, undertake a focus group which identified positive changes in practice and commitment to professional development recognise the need to extend the programme's evaluation to stakeholders (Kirkpatrick's level 4) 